\title{
Phase Diagram and Edge States of Surface States of Topological Superconductors
}

\author{
Weihao Zhao \\ College of Science, University of Shanghai for Science and Technology, Shanghai, China \\ Email: 15230818987@163.com
}

How to cite this paper: Zhao, W.H. (2021) Phase Diagram and Edge States of Surface States of Topological Superconductors. World Journal of Condensed Matter Physics, 11, 65-76.

https://doi.org/10.4236/wjcmp.2021.113005

Received: July 2, 2021

Accepted: August 24, 2021

Published: August 27, 2021

Copyright $\odot 2021$ by author(s) and Scientific Research Publishing Inc. This work is licensed under the Creative Commons Attribution International License (CC BY 4.0).

http://creativecommons.org/licenses/by/4.0/

\section{(c) (i) Open Access}

\begin{abstract}
Majorana fermions in two-dimensional systems satisfy non-Abelian statistics. They are possible to exist in topological superconductors as quasi particles, which is of great significance for topological quantum computing. In this paper, we study a new promising system of superconducting topological surface state topological insulator thin films. We also study the phase diagrams of the model by plotting the Majorana edge states and the density of states in different regions of the phase diagram. Due to the mirror symmetry of the topological surface states, the Hamiltonian can be block diagonalized into two spin-triplet $p$-wave superconductors, which are also confirmed by the phase diagrams. The chiral Majorana edge modes may provide a new route for realizing topological quantum computation.
\end{abstract}

\section{Keywords}

Majorana Fermion, Phase Diagrams, Topological Superconductor, Surface States

\section{Introduction}

In 1937, Ettore Majorana [1] proposed the existence of a type of fermion, known as Majorana fermion, which is its own antiparticle [2] [3]. Since the Majorana fermions were proposed, people have been looking for them [4] [5] [6]. The most representative one is the p-wave superconductor, which is influenced by the Moore-Read fractional quantum Hall (Pfaffian) state [7]. Because of the nontrivial topology of bulk Chern number, there are chiral Majorana fermion edge modes trapped in the vortices in the 2-dimensional $p$-wave superconductor [8]. Because of the non-abelian weaving of Majorana fermions, topological quantum computation can be realized based on them. For example, topological quantum computing can be achieved by using a non-abelian topological order containing the Ising 
non-abelian arbitrarily [9]. Due to the great application prospect of topological quantum computing, people have spent a lot of energy to find the substance that can satisfy the p-wave superconductor, among which the most studied substance is $\mathrm{Sr}_{2} \mathrm{RuO}_{4}$ [10] [11] [12]. However, the discussion of $\mathrm{Sr}_{2} \mathrm{RuO}_{4}$ is more of a stay in the theoretical discussion, and no definite conclusion has been reached [13] [14]. In the case of spin-orbit interaction, the conventional s-wave superconducting state may also support topological phase. However, the quantum anomalous Hall insulator can transform into a two-dimensional chiral topological superconducting phase driven by the $s$-wave superconducting proximity effect. In principle, topological quantum computation based on manual Majorana edge modules can be realized [15] [16] [17]. Recent research is mainly focused on $\mathrm{Fe}$ (Te, Se) as the representative of the iron-based superconductivity [18] [19].

Under normal states, iron-based materials with small Fermi levels are considered topological metals with slightly doped topological surface states, which can be observed by the angular resolved spectrum of spin polarization [20] [21]. The study of angular resolved spectrum shows that, due to the influence of volume superconductivity, a superconducting energy gap similar to the energy band gap will be generated on the topological surface states of a fully gap superconductor with temperature below $T_{O}$ which is the topological superconducting surface states [21]. Topological surface state superconductivity is proposed by Fu-Kane [5], which is realized by inducing superconductivity in topological surface states of strong topological insulators by $s$-wave superconductivity proximity effect. In the absence of an external magnetic field, the Majorana zero model can be observed in the gap of $\mathrm{FeTe}_{0.55} \mathrm{Se}_{0.45}$ and in the quantum anomalous vortices nucleated at Fe site [22] [23] [24]. Based on the above research progress, we will discuss below the chiral topological superconductor used to generate nonmagnetic two-dimensional time-inversion symmetry breaking in topological surface state superconductivity, and construct a superconducting topological surface state coupling model on the upper and lower surfaces of the film, as shown in Figure 1(a). Or construct a coupled model of two topological surface state superconductors with opposite surfaces as shown in Figure 1(b). Both models are based on iron-based superconductors.

(a)

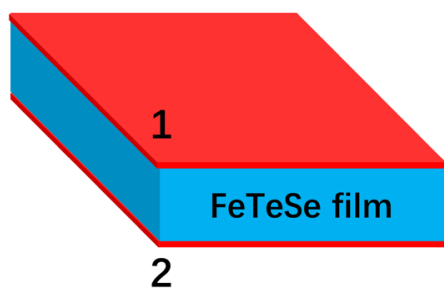

(b)

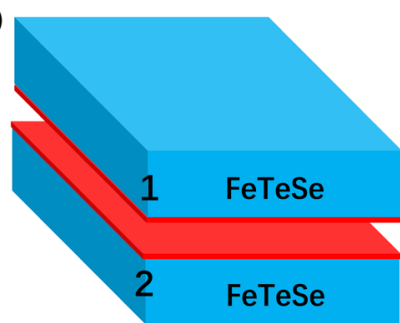

Figure 1. The coupled superconducting topological surface states illustrated with FeTeSe. The red area represents the surface states and the blue area represents the body states. (a) Indicates that the top and bottom of the FeTeSe film have topological superconducting surface states. (b) Represents two topological surface state superconductors having opposite surfaces. 


\section{Model Hamiltonian}

First, we need to construct the Hamiltonian of the model, which is selected based on Nambu basis: $\Psi_{N}=\left(\psi_{1 \uparrow q}, \psi_{1 \downarrow q}, \psi_{2 \uparrow q}, \psi_{2 \downarrow q}, \psi_{1 \uparrow-q}^{\dagger}, \psi_{1 \downarrow-q}^{\dagger}, \psi_{2 \uparrow-q}^{\dagger}, \psi_{2 \downarrow-q}^{\dagger}\right)^{\mathrm{T}}, 1$ and 2 represent the surface states of the upper and lower surfaces respectively, and these two indexes represent the pseudo spin states in the spin-orbit coupling respectively, and $\mathrm{q}$ index represents the momentum, the Fermi velocity is set as 1. With the basis vector selected, the corresponding Bogoliubov-de Gennes (BdG) [8] Hamiltonian is:

$$
H(\boldsymbol{q})=\left(\begin{array}{cc}
h_{x}(\boldsymbol{q}) & \Delta_{x}^{\dagger} \\
\Delta_{x} & h_{x}^{*}(-\boldsymbol{q})
\end{array}\right)
$$

$h_{x}(\boldsymbol{q})$ and $\Delta_{x}$ are expressed as follows:

$$
\begin{gathered}
h_{x}(\boldsymbol{q})=\left(\begin{array}{cccc}
\lambda-\mu & q_{1}-i q_{2} & t & 0 \\
q_{1}+i q_{2} & -\lambda-\mu & 0 & t \\
t & 0 & \lambda-\mu & -q_{1}+i q_{2} \\
0 & t & -q_{1}-i q_{2} & -\lambda-\mu
\end{array}\right) \\
\Delta_{x}=\left(\begin{array}{cccc}
\Delta & 0 & -\Delta_{s}-\Delta_{t 1}-i \Delta_{t 2} \\
0 & \Delta_{1} & \Delta_{s}-\Delta_{t 1}-i \Delta_{t 2} & 0 \\
-\Delta_{1} & 0 & 0 & -\Delta_{2} \\
0 & -\Delta_{s}+\Delta_{t 1}+i \Delta_{t 2} & \Delta_{2} & 0
\end{array}\right)
\end{gathered}
$$

We make a substitution such that $\alpha=\frac{\Delta_{1}+\Delta_{2}}{2}$ and $\beta=\frac{\Delta_{1}-\Delta_{2}}{2}$, so that $\Delta_{1}$ and $\Delta_{2}$ can be represented correspondently in terms of $\alpha$ and $\beta$ as $\Delta_{1}=\alpha+\beta$ and $\Delta_{2}=\alpha-\beta$. After the above substitution, the Hamiltonian of our model will be as follows:

$$
\begin{aligned}
H_{1}= & q_{1} \sigma_{x} \chi_{z} \tau_{0}+q_{2} \sigma_{y} \chi_{z} \tau_{z}-\mu \sigma_{0} \chi_{0} \tau_{z}+\lambda \sigma_{z} \chi_{x} \tau_{z}+t \sigma_{0} \chi_{x} \tau_{z} \\
& -\Delta_{s} \sigma_{y} \chi_{x} \tau_{y}+\frac{\alpha}{2} \sigma_{y} \chi_{z} \tau_{y}+\frac{\beta}{2} \sigma_{y} \chi_{0} \tau_{y} \\
& -\Delta_{t 1} \sigma_{x} \chi_{y} \tau_{y}+\Delta_{t 2} \sigma_{x} \chi_{y} \tau_{x}
\end{aligned}
$$

Define a mirror symmetry $\mathcal{M}_{-}=-i \sigma_{z} \chi_{x} \tau_{0}$, then change the above Hamiltonian $H_{1}$ into $\mathcal{M}_{-} H_{1} \mathcal{M}_{-}^{\mathrm{T}}$ under the action of mirror symmetry $\mathcal{M}_{-}$, and the result after change is as follows:

$$
\begin{aligned}
\mathcal{M}_{-} H_{1} \mathcal{M}_{-}^{\mathrm{T}}= & -q_{1} \sigma_{x} \chi_{z} \tau_{0}-q_{2} \sigma_{y} \chi_{z} \tau_{z}+\mu \sigma_{0} \chi_{0} \tau_{z}+\lambda \sigma_{z} \chi_{x} \tau_{z}-t \sigma_{0} \chi_{x} \tau_{z} \\
& -\Delta_{s} \sigma_{y} \chi_{x} \tau_{y}-\frac{\alpha}{2} \sigma_{y} \chi_{z} \tau_{y}+\frac{\beta}{2} \sigma_{y} \chi_{0} \tau_{y} \\
& +\Delta_{t 1} \sigma_{x} \chi_{y} \tau_{y}-\Delta_{t 2} \sigma_{x} \chi_{y} \tau_{x}
\end{aligned}
$$

By comparing Equations (4) and (5), we can get $\alpha, \Delta_{t 1}$ and $\Delta_{t 2}$ are odd symmetry of the mirror image about $\mathcal{M}_{-}$, while the term $\beta$ is even symmetry of the mirror image about $\mathcal{M}_{-}$. When $\Delta_{t 1}, t, \Delta_{1}$ and $\Delta_{2}$ are all non-zero, the time inversion symmetry of Hamiltonian described in Equation (1) will spontaneously break, and only when the time inversion symmetry is broken, will there be a non-zero Chern number. 


\section{Topological Phase Diagram}

Figure 2 describes the phase diagram of the relationship between the real part $\Delta_{t 1}$ and the imaginary part $\Delta_{t 2}$ of Hamiltonian $\mathcal{M}_{-} H_{1} \mathcal{M}_{-}^{\mathrm{T}}$ described in Equation (5). The selection of specific parameters is shown in the figure, and $\mathrm{N}$ represents the Chern number of each region.

Figure 2 depicts the phase diagram of Hamiltonian $\mathcal{M}_{-} H_{1} \mathcal{M}_{-}^{\mathrm{T}}$ in the $\Delta_{t 1}-\Delta_{t 2}$ plane. Next, $\Delta_{t 1}+i \Delta_{t 2}$ is expressed in exponential form, that is, $-\theta e^{-i \phi}$ is used to replace $\Delta_{t 1}+i \Delta_{t 2}$. The phase diagram between its module value $\theta$ and phase Angle $\phi$ is shown in Figure 3. The influence of the upper and lower surfaces on the phase diagram is not considered here, so the parameters of the upper and lower surfaces are set to be the same here, and their sizes

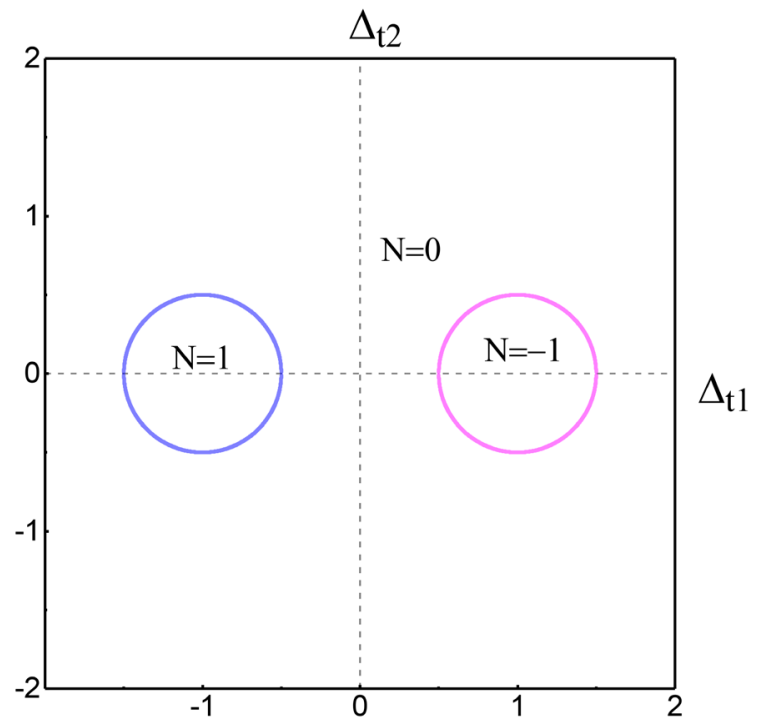

Figure 2. The topological phase diagram of Hamiltonian $\mathcal{M}_{-} H_{1} \mathcal{M}_{-}^{\mathrm{T}}$ in the $\Delta_{t 1}-\Delta_{t 2}$ plane for $\mu=0, t=0.5, \alpha=1$ and $\beta=0$.

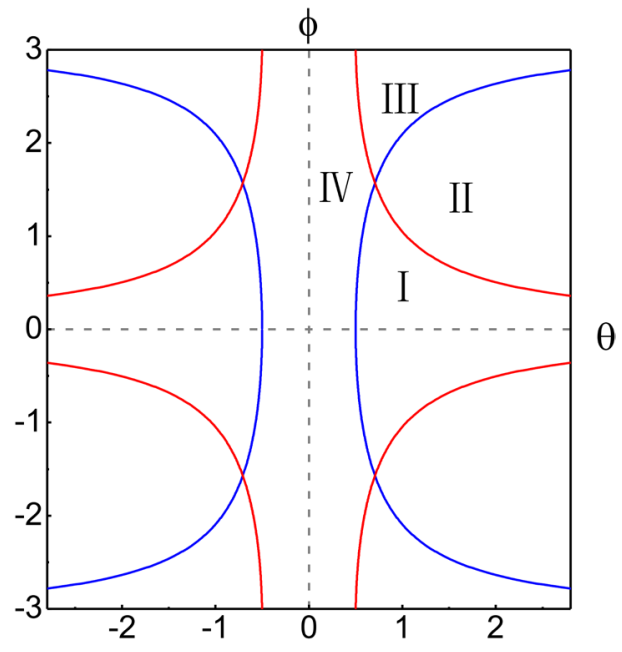

Figure 3. The topological phase diagram of Hamiltonian $H$ in the $\theta-\phi$ plane for $\mu=0, \lambda=0, q_{1}=0, q_{2}=0, \quad t=1, \Delta_{s}=0, \Delta_{1}=\Delta_{2}=\theta$. 
are set to be the same as the module of the complex number $\Delta_{t 1}+i \Delta_{t 2}$, that is $\Delta_{1}=\Delta_{2}=\theta$. As can be seen from Figure 3, the figure is symmetric about the coordinate axis, so we mainly discuss the Chern number, edge states and their density of states in the first quadrant. In Figure 3, there are four regions in the first quadrant, and each region is separated by solid lines, where solid lines represent phase transition points and topological phase transition will occur after passing through the solid lines. By calculating (see Appendix), it can be obtained that the Chern number of the region I is -1 , the Chern number of the region III is +1 , and the Chern number of the region II and region IV are both 0 . To further understand the distribution of the densities in each region, a unitary transformation can be defined:

$$
U_{-}=\frac{1}{\sqrt{2}}\left(\begin{array}{cccccccc}
0 & 0 & 0 & 0 & 1 & 0 & 1 & 0 \\
0 & 1 & 0 & -1 & 0 & 0 & 0 & 0 \\
1 & 0 & 1 & 0 & 0 & 0 & 0 & 0 \\
0 & 0 & 0 & 0 & 0 & 1 & 0 & -1 \\
1 & 0 & -1 & 0 & 0 & 0 & 0 & 0 \\
0 & 0 & 0 & 0 & 0 & 1 & 0 & 1 \\
0 & 0 & 0 & 0 & 1 & 0 & -1 & 0 \\
0 & 1 & 0 & 1 & 0 & 0 & 0 & 0
\end{array}\right)
$$

If the unitary operator $U_{-}$is applied to the Hamiltonian $\mathrm{H}$, then the Hamiltonian becomes a block diagonal matrix under the action of the unitary operator by considering the case of $\Delta_{1}=\Delta_{2}=\Delta=\theta$ :

$$
\begin{gathered}
U_{-} H U_{-}^{-1}=\left(\begin{array}{cc}
h_{1} & 0 \\
0 & h_{2}
\end{array}\right) \\
h_{1}=\left(\begin{array}{cccc}
-t-\lambda+\mu & -\theta-\theta \mathrm{e}^{-i \phi} & 0 & q_{1}-i q_{2} \\
-\theta-\theta \mathrm{e}^{i \phi} & -t-\lambda-\mu & q_{1}-i q_{2} & 0 \\
0 & q_{1}+i q_{2} & t+\lambda-\mu & \theta+\theta \mathrm{e}^{i \phi} \\
q_{1}+i q_{2} & 0 & \theta+\theta \mathrm{e}^{-i \phi} & t+\lambda+\mu
\end{array}\right) \\
h_{2}=\left(\begin{array}{cccc}
-t+\lambda-\mu & \theta-\theta \mathrm{e}^{i \phi} & 0 & q_{1}+i q_{2} \\
\theta-\theta \mathrm{e}^{-i \phi} & -t+\lambda+\mu & q_{1}+i q_{2} & 0 \\
0 & q_{1}-i q_{2} & t-\lambda+\mu & -\theta+\theta \mathrm{e}^{-i \phi} \\
q_{1}-i q_{2} & 0 & -\theta+\theta \mathrm{e}^{i \phi} & t-\lambda-\mu
\end{array}\right)
\end{gathered}
$$

Next, the corresponding phase diagram of $h_{1}$ in the $\theta-\phi$ plane is drawn, as shown in Figure 4(a). Figure 4(a) is also symmetric about the coordinate axis. In the first quadrant of the figure, there are two regions, in which the Chern number of the region $I$ is -1 and the Chern number of the region II is 0 . We have given the phase diagram corresponding to $h_{2}$, as shown in Figure 4(b). The selection of parameters in this diagram is the same as that in the phase diagram drawn by $h_{1}$. There are also two regions in the first quadrant of (b) in Figure 4, where the Chern number of the region $I$ is +1 , and the positive stale number of the region II is 0 . By comparing Figure 3 and Figure 4, it can be 
(a)

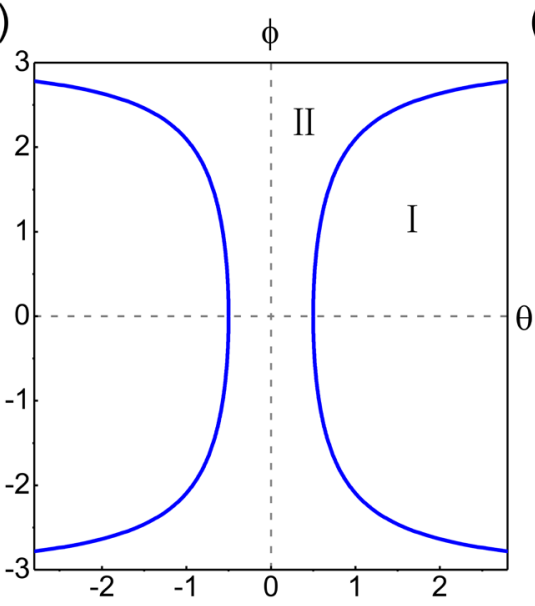

(b)

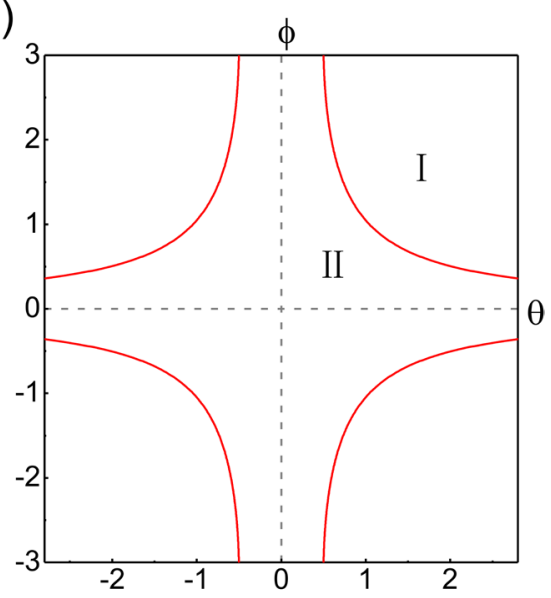

Figure 4. The topological phase diagram of Hamiltonian (a) $h_{1}$ and (b) $h_{2}$ in the $\theta-\phi$ plane for $\mu=0, \lambda=0, q_{1}=0, q_{2}=0, t=1, \Delta_{s}=0$.

found that the phase diagram described by the original Hamiltonian is exactly equal to the superposition of the two-phase diagrams described by the block matrix obtained after the unitary transformation.

\section{Edge States and State Density}

\subsection{Discussion on Edge States}

Next, edge states and their state density diagrams are discussed. The BdG Hamiltonian can be written as follows in the case of Nambu basis:

$$
\mathcal{H}_{B d G}=\sum_{q} \Psi_{N}^{\dagger} H(\boldsymbol{q}) \Psi_{N} \text {. }
$$

In the lattice model, Hamiltonian variables are replaced as follows:

$t \rightarrow t_{0}+t_{1}\left(q_{1}^{2}+q_{2}^{2}\right), \quad q_{2} \rightarrow a^{-1} \sin \left(q_{2} a\right)$ and $q_{2}^{2} \rightarrow 2\left(1-a^{-2} \cos \left(q_{2} a\right)\right)$ Where a represents the lattice constant, which can be taken as 1 , and the direction $q_{2}$ is the open edge condition. First, the edge states of each region in Figure 3 in the $q_{1}-E$ plane are drawn. The edge state graph of the corresponding point in the region I in Figure 3 is shown in Figure 5(a), in this figure the red (blue) line stands for the spectrum of the chiral edge mode localized on the right (left) side of the sample (see Figure 6(a)). From Figure 5(a), it can be found that there is an intersection point within the small range of coordinate $(0,0)$, as shown in the red line and blue line in the figure, the two lines are the gapless edge states. The red line and the blue line will only travel along the edge, and the direction of the electrons will only travel in a certain direction. The selection of parameters in Figure 5(a) corresponds to the I region in Figure 3, and the Chern number of this region can be calculated as: $N=-1$, and its absolute value is 1 , corresponding to edge states in Figure 5.

Next, the edge states of points corresponding to the region II in Figure 3 are plotted, as shown in Figure 5(b), in this figure the red and green (blue and orange) lines stand for the spectrum of the chiral edge mode localized on the left (right) side of the sample (see Figure 6(b)). By comparing Figure 5(a) and 

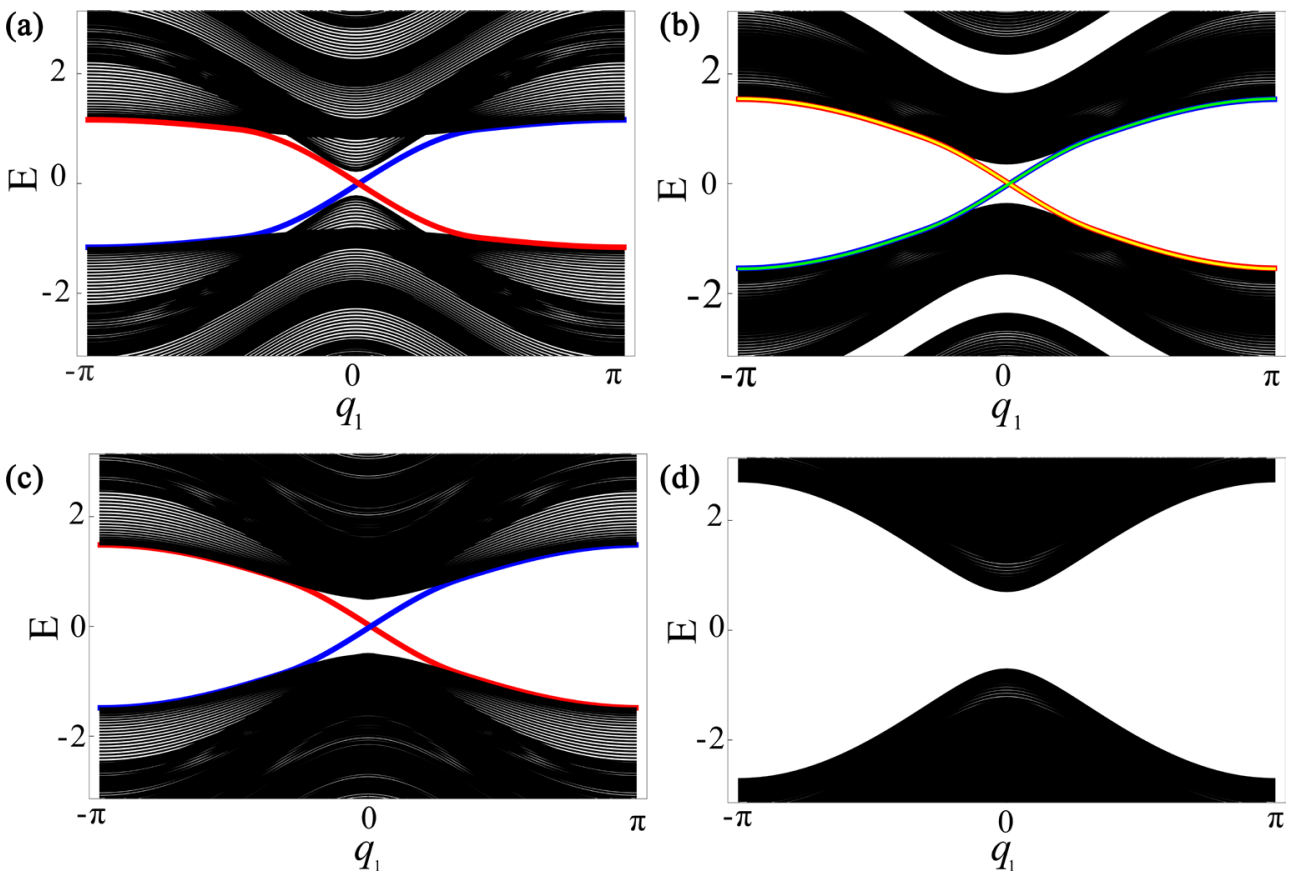

Figure 5. Edge state graphs with different parameters for $t_{0}=1.0, t_{1}=1.0, \mu=0, \Delta_{s}=0$, $\lambda=0$, (a) $\theta=1.0, \phi=0.8$. (b) $\theta=1.0, \phi=1.5$. (c) $\theta=0.8, \phi=2.5$. (d) $\theta=0.2, \phi=1.5$.

Figure 5(b) can be found: in Figure 5(b) edge of the graphics in the coordinates of point $(0,0)$ is, in fact, there are two near the intersection, but the edge of the two is overlap together, in order to show the difference between the edge of the two groups, we use lines of different colors to distinguish, the line color is respectively: In red and yellow, the upward lines are blue and green, respectively. The parameters selected in Figure 5(b) correspond to the region II in Figure 3, so the Chern number of this region is calculated as $N=0$. Edge states of Figure 5(b) is two, but the area of the Chern number is 0 , that does not conform to the body and the edge of the correspondence. In order to explain the phenomenon, we make a unitary operator $U_{-}$on the Hamiltonian $H(\boldsymbol{q})$, let it become a block diagonal matrix, the matrix $h_{1}$ and $h_{2}$ respectively, and the two pieces of these two pieces of matrix of the phase diagram in Figure 4, respectively (a) and (b) figure. In Figure 4(a), the Chern number of I region is -1 , and the Chern number of II region in Figure 4(b) is 1, which exactly corresponds to the Chern number of I in the region in Figure 3, $N=1+(-1)=0$, which is the reason why the Chern number of the region in Figure 3 is 0 . In the area of Figure 3 II Chen number 0 is not $0+0=0$, but rather the result of 1 and -1 in Figure 5(b) there will be two edge, one of the representative $h_{1}$ describes the edge of the Chern number of 1 , the other is $h_{2}$ describes the edge of the corresponding Chern number is 1 .

The edge state of the corresponding point in the region III in Figure 3 is shown in Figure 5(c), in this figure the red (blue) line stands for the spectrum of the chiral edge mode localized on the left (right) side of the sample (see Figure 6(c)). Contrast Figure 5(b) and Figure 5(c) can be found that the Chern num- 
ber of the edge of graph into a group, which means in Figure 5(b) and Figure 5 (c) between two groups of parameters will be after a topological phase transition point, it is because the topological transformation happened, so will lead to the edge of the area III in Figure 3 state is only a set, the topological transformation point is Figure 3 part within the solid line.

Finally, the edge state graph of the corresponding point in the region IV in Figure 3 is drawn, as shown in Figure 5(d). As can be seen in Figure 5(d), there is no intersection point at $(0,0)$. Therefore, it can be shown that under the parameter selection of this region, the Hamiltonian model does not have an edge state, but a full gap. In Figure 3 can be obtained by calculation IV area Chern number is 0 , Chen and Chen several of the region just correspond to Figure 4(a) graph area II and (b) graph area II, these two areas and the corresponding Chern number is 0 , so Figure 3 of IV area corresponds to the Chern number for $N=0$ $+0=0$, so the edge of the area is there exists gap, which further illustrates the body-edge correspondence.

\subsection{Density Distribution of States in Real Space of Wave Function}

In order to further explain the distribution of wave function in real space in each group of edge state graphs, we draw density distribution of states with different parameters selected, and select the number of lattice points in the $y$ direction as $y=50$ in each graph drawing.

In the edge state graph corresponding to Figure 5(a), when the value of $q_{1}$ is 0.2 , the real space state density distribution diagram of wave function corresponding to points on the marginal state is shown in Figure 6(a), and the parameters are selected as shown in the figure. In Figure 6(a), the density of wave function state represented by different colors corresponds to the different edge states in Figure 5(a), and it can be found that the density of wave function state of the two edge states is localized at both ends respectively.

Figure 6(b) presents the real space state density distribution corresponding to the two groups of edge states when $q_{1}=0.2$ under the same parameter selection as Figure 5(b). Energy state density represented by different colors correspond to

(a)

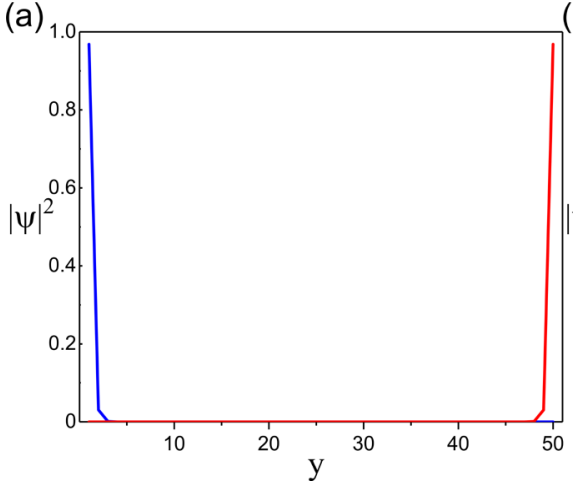

(b)

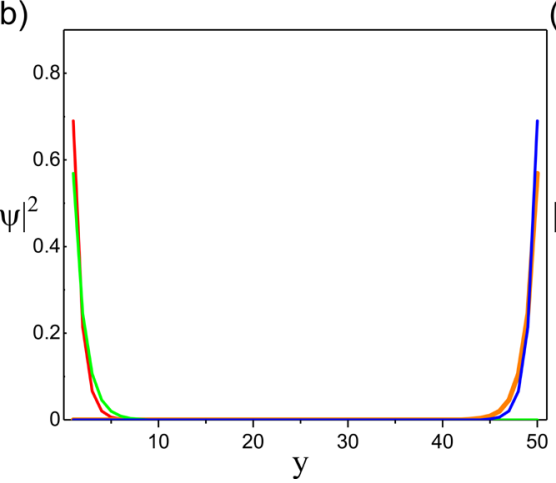

(c)

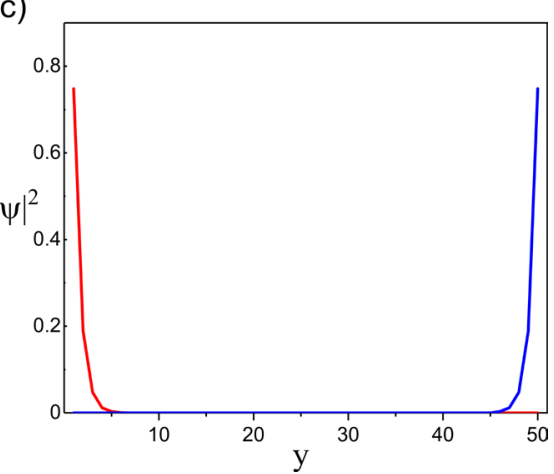

Figure 6. Real space state density distribution of wave function under different parameters for $t_{0}=1.0, t_{1}=1.0, \mu=0, \Delta_{s}=0$, $\lambda=0, q_{1}=0.2$. (a) $\theta=1.0, \phi=0.8$. (b) $\theta=1.0, \phi=1.5$. (c) $\theta=0.8, \phi=2.5$. 
points on edge states of different colors. We can see that for the density of states of the points on the edge states with the same positive and negative slope, they will be localized at both ends, which can indicate that the Chern number of the II region in Figure 3 is the structure of $(N=1) \oplus(N=-1)$. For two symmetrical Density of States diagrams, they correspond to the same pattern.

Figure $6(\mathrm{c})$ corresponds to the density distribution of real space states corresponding to the points on the edge state when $q_{1}=0.2$ in the group of edge state graphs in Figure 5(c). By comparing Figure 6(c) with Figure 6(a), it can be found that when the slope is positive, the density distribution of real space states corresponding to points on the edge states will be localized at opposite edges. This is because Figure 6(a) corresponds to the region with a Chern number of -1 in Figure 3, while Figure 6(c) corresponds to the region with a Chern number of 1 in Figure 3, indicating that the Chern number will affect the number of edge states. Although the positive and negative Chern number will not affect the number of edge states, it will affect the distribution of the energy state density of the wave function.

\section{Conclusion}

In this paper, we study the phase diagram, edge state and density distribution diagram of the topological surface state superconducting in detail. It is found that the phase diagram of Hamiltonian $H(\boldsymbol{q})$ is the same as the superposition of the phase diagram among the block matrices obtained by the Hamiltonian after the unitary transformation of $U_{-}$. In nontrivial topological phase, the corresponding phase diagrams are drawn by discussing the lattice model, and the edge states of different regions in the phase diagrams are also given, the difference between the corresponding wave function in the real space distribution state density diagram, thus further proving that Majorana zero model of the number is the same with the body of Chern number, body-edge correspondence. In the edge state, the electron propagates in a certain direction and does not backscatter. Because the Majorana zero model can well solve the problems caused by quantum decoherence, and the Majorana fermions obey non-abelian statistics, the encoding problem of quantum information can be solved by using the Majorana fermions, which will have great application value in the development of topological quantum computing.

\section{Acknowledgements}

We thank Xi Luo for helpful discussions. This work is supported by NNSF of China with No. 11804223.

\section{Conflicts of Interest}

The author declares no conflicts of interest regarding the publication of this paper.

\section{References}

[1] Majorana, E. (1937) Teoria simmetrica dell'elettrone e del positrone. Il Nuovo Ci- 
mento (1924-1942), 14, Article No. 171. https://doi.org/10.1007/BF02961314

[2] Akhmerov, A.R., Nilsson, J. and Beenakker, C.W.J. (2009) Electrically Detected Interferometry of Majorana Fermions in a Topological Insulator. Physical Review Letters, 102, Article ID: 216404. https://doi.org/10.1103/PhysRevLett.102.216404

[3] Sato, M. and Fujimoto, S. (2010) Existence of Majorana Fermions and Topological Order in Nodal Superconductors with Spin-Orbit Interactions in External Magnetic Fields. Physical Review Letters, 105, Article ID: 217001. https://doi.org/10.1103/PhysRevLett.105.217001

[4] Alicea, J. (2012) New Directions in the Pursuit of Majorana Fermions in Solid State Systems. Reports on Progress in Physics, 75, Article ID: 076501. https://doi.org/10.1088/0034-4885/75/7/076501

[5] Fu, L. and Kane, C.L. (2008) Superconducting Proximity Effect and Majorana Fermions at the Surface of a Topological Insulator. Physical Review Letters, 100, Article ID: 096407. https://doi.org/10.1103/PhysRevLett.100.096407

[6] Lutchyn, R.M., Sau, J.D. and Sarma, D. (2010) Majorana Fermions and a Topological Phase Transition in Semiconductor-Superconductor Heterostructures. Physical Review Letters, 105, Article ID: 077001.

https://doi.org/10.1103/PhysRevLett.105.077001

[7] Moore, G. and Read, N. (1991) Nonabelions in the Fractional Quantum Hall Effect. Nuclear Physics B, 360, 362-396. https://doi.org/10.1016/0550-3213(91)90407-O

[8] Read, N. and Green, D. (2000) Paired States of Fermions in Two-Dimensions with Breaking of Parity and Time Reversal Symmetries, and the Fractional Quantum Hall Effect. Physical Review B, 61, 10267-10297. https://doi.org/10.1103/PhysRevB.61.10267

[9] Wen, X.G. (1991) Non-Abelian Statistics in the Fractional Quantum Hall States. Physical Review Letters, 66, 802-805. https://doi.org/10.1103/PhysRevLett.66.802

[10] Mackenzie, A.P. and Maeno, Y. (2003) The Superconductivity of $\mathrm{Sr}_{2} \mathrm{RuO}_{4}$ and the Physics of Spin-Triplet Pairing. Reviews of Modern Physics, 75, 657-712. https://doi.org/10.1103/RevModPhys.75.657

[11] Ueno, Y., Yamakage, A., Tanaka, Y. and Sato, M. (2013) Symmetry-Protected Majorana Fermions in Topological Crystalline Superconductors: Theory and Application to $\mathrm{Sr}_{2} \mathrm{RuO}_{4}$. Physical Review Letters, 111, Article ID: 087002. https://doi.org/10.1103/PhysRevLett.111.087002

[12] Maeno, Y., Kittaka, S. and Nomura, T. (2012) Evaluation of Spin-Triplet Superconductivity in $\mathrm{Sr}_{2} \mathrm{RuO}_{4}$. Journal of the Physical Society of Japan, 81, Article ID: 011009. https://doi.org/10.1143/JPSJ.81.011009

[13] Rice, T.M. and Sigrist, M. (1995) $\mathrm{Sr}_{2} \mathrm{RuO}_{4}$ : An Electronic Analogue of ${ }^{3} \mathrm{He}$. Journal of Physics: Condensed Matter, 7, Article No. L643.

https://doi.org/10.1088/0953-8984/7/47/002

[14] Pustogow, A., Luo, Y.K., Chronister, A., Su, Y.-S., Sokolov, D.A., Jerzembeck, F., et al. (2019) Constraints on the Superconducting Order Parameter in $\mathrm{Sr}_{2} \mathrm{RuO}_{4}$ from Oxygen-17 Nuclear Magnetic Resonance. Nature, 574, 72-75. https://doi.org/10.1038/s41586-019-1596-2

[15] Qi, X.L., Hughes, T.L. and Zhang, S.C. (2010) Chiral Topological Superconductor from the Quantum Hall State. Physical Review B, 82, Article ID: 184516. https://doi.org/10.1103/PhysRevB.82.184516

[16] He, Q.L., Pan, L., Stern, A.L., Burks, E.C., Che, X., Yin, G., et al. (2017) Chiral Majorana Fermion Modes in a Quantum Anomalous Hall Insulator-Superconductor 
Structure. Science, 357, 294-299. https://doi.org/10.1126/science.aag2792

[17] Lian, B., Sun, X.Q. and Vaezi, A. (2018) Topological Quantum Computation Based on Chiral Majorana Fermions. Proceedings of the National Academy of Sciences of the United States of America, 115, 10938-10942.

https://doi.org/10.1073/pnas.1810003115

[18] Kong, L.Y., Zhu, S.Y., Papaj, M., Chen, H., Cao, L., Isobe, H., et al. (2019) Half-Integer Level Shift of Vortex Bound States in an Iron-Based Superconductor. Nature Physics, 15, 1181-1187. https://doi.org/10.1038/s41567-019-0630-5

[19] Wang, Z.Y., Rodriguez, J.O., Jiao, L., Howard, S., Graham, M., Gu, G.D., et al. (2020) Evidence for dispersing 1D Majorana Channels in an Iron-Based Superconductor. Science, 367, 104-108. https://doi.org/10.1126/science.aaw8419

[20] Zhang, P., Wang, Z., Wu, X., Yaji, K., Ishida, Y., Kohama, Y., et al. (2019) Multiple Topological States in Iron-Based Superconductors. Nature Physics, 15, 41-47. https://doi.org/10.1038/s41567-018-0280-Z

[21] Zhang, P., Yaji, K., Hashimoto, T., Ota, Y., Kondo, T., Okazaki, K., et al. (2018) Observation of Topological Superconductivity on the Surface of an Iron-Based Superconductor. Science, 360, 182-186. https://doi.org/10.1126/science.aan4596

[22] Wang, D.F., Kong, L.Y., Fan, P., Chen, H., Zhu, S., Liu, W., et al. (2018) Evidence for Majorana Bound States in an Iron-Based Superconductor. Science, 362, 333-365. https://doi.org/10.1126/science.aao1797

[23] Liu, Q., Chen, C., Zhang, T., Peng, R., Yan, Y.-J., Wen, C.-H.-P., et al. (2018) Robust and Clean Majorana Zero Mode in the Vortex Core of High-Temperature Superconductor $\left(\mathrm{Li}_{0.84} \mathrm{Fe}_{0.16}\right)$ OHFeSe. Physical Review X, 8, Article ID: 041056. https://doi.org/10.1103/PhysRevX.8.041056

[24] Yin, J.X., Wu, Z., Wang, J.H., Ye, Z.-Y., Gong, J., Hou, X.-Y., et al. (2015) Observation of a Robust Zero-Energy Bound State in Iron-Based Superconductor Fe(Te,Se). Nature Physics, 11, 543-546. https://doi.org/10.1038/nphys3371

[25] Fukui, T., Hatsugai, Y. and Suzuki, H. (2005) Chern Numbers in Discretized Brillouin Zone: Efficient Method of Computing (Spin) Hall Conductances. Journal of the Physical Society of Japan, 74, 1674-1677. https://doi.org/10.1143/JPSJ.74.1674 


\section{Appendix}

In a two-dimensional system, it is assumed that a point $k_{l}$ in the discrete Brillouin region can be expressed as [25]:

$$
\begin{gathered}
k_{l}=\left(k_{j 1}, k_{j 2}\right) \\
k_{j_{\mu}}=\frac{2 \pi j_{\mu}}{q_{\mu} N_{\mu}},\left(j_{\mu}=0, \cdots, N_{\mu}-1\right)
\end{gathered}
$$

$\left|n\left(k_{l}\right)\right\rangle$ is periodic on the lattice. First define the Chern number of the nth band as:

$$
\tilde{c}_{n} \equiv \frac{1}{2 \pi i} \sum_{l} \tilde{F}_{12}\left(k_{l}\right)
$$

For the wave function of the nth band, a connection variable can be defined:

$$
U_{\mu}\left(k_{l}\right) \equiv\left\langle n\left(k_{l}\right) \mid n\left(k_{l}+\hat{\mu}\right)\right\rangle / \mathcal{N}_{\mu}\left(k_{l}\right)
$$

where $\mathcal{N}_{\mu}\left(k_{l}\right) \equiv\left|\left\langle n\left(k_{l}\right) \mid n\left(k_{l}+\hat{\mu}\right)\right\rangle\right|$. Lattice strength is defined as

$$
\tilde{F}_{12}\left(k_{l}\right) \equiv \ln U_{1}\left(k_{l}\right) U_{2}\left(k_{l}+\hat{1}\right) U_{1}\left(k_{l}+\hat{2}\right)^{-1} U_{2}\left(k_{l}\right)^{-1}
$$

Since $\tilde{c}_{n}$ is gauge invariant, the gauge potential energy is selected as:

$$
\tilde{A}_{\mu}\left(k_{l}\right)=\ln U_{\mu}\left(k_{l}\right),-\pi<\frac{1}{i} \tilde{A}_{\mu}\left(k_{l}\right) \leq \pi
$$

which is periodic on the lattice: $\tilde{A}_{\mu}\left(k_{l}+N_{\mu} \hat{\mu}\right)=\tilde{A}_{\mu}\left(k_{l}\right)$. Under the selection of the gauge potential, $\tilde{F}_{12}\left(k_{l}\right)$ becomes:

$$
\tilde{F}_{12}\left(k_{l}\right)=\Delta_{1} \tilde{A}_{2}\left(k_{l}\right)-\Delta_{2} \tilde{A}_{1}\left(k_{l}\right)+2 \pi i n_{12}\left(k_{l}\right)
$$

where $\Delta_{\mu}$ can be expressed as: $\Delta_{\mu} f\left(k_{l}\right)=f\left(k_{l}+\hat{\mu}\right)-f\left(k_{l}\right)$ and $n_{12}\left(k_{l}\right)$ is an integer valued field. So we can get the lattice Chern number:

$$
\tilde{c}_{n}=\sum_{l} n_{12}\left(k_{l}\right)
$$

